\title{
鹖pubvet
}

https://doi.org/10.31533/pubvet.v12n7a133.1-6

\section{Neuroblastoma olfatório em cão: achados tomográficos e histopatológicos}

Lourival Barros de Sousa Brito Pereira ${ }^{1}$, Homero Firmo Pessoa ${ }^{2}$, Lucilo Bioni da Fonseca Filho $^{3 * 0}$, Andréa Jullyanna de Carvalho ${ }^{40}$, Melissa Barbosa Pontes ${ }^{50}$, Nicolli de Albuquerque Leal Gomes D'Alcantara ${ }^{6}{ }^{\bullet}$, Jordy Diniz de Oliveira Lima ${ }^{7} \bullet$, Priscilla Virginio de Albuquerque $^{8 \odot}$, Júlio Cézar dos Santos Nascimento ${ }^{\ominus} \odot$, Wagner McKlayton Alves de Souza ${ }^{10} \odot$

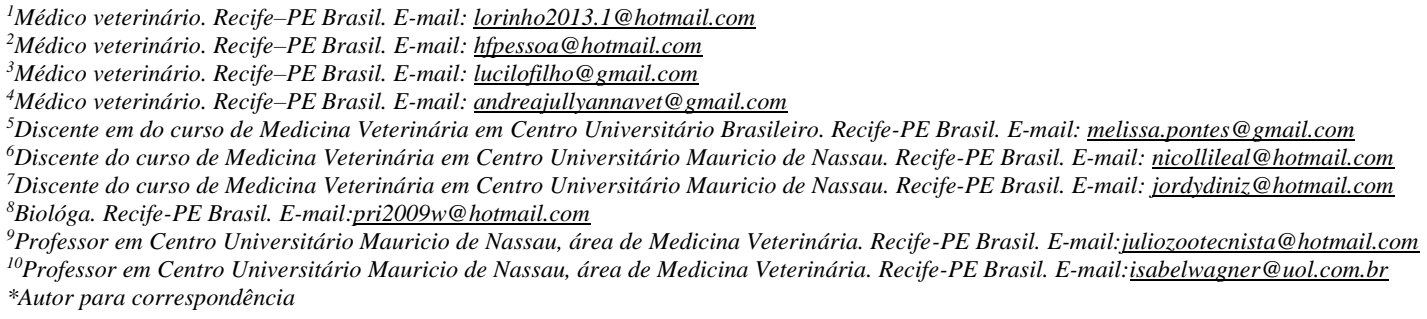

RESUMO. As ocorrências de neoplasias na cavidade nasal em cães ocorrem em aproximadamente $1 \%$ de todas as neoplasias. O neuroblastoma olfatório é uma neoplasia neuroepitelial da cavidade nasal e seios paranasais raramente relatada em cães e tem como principais sinais clínicos epistaxe, espirros, dificuldade respiratória, deformidade facial e alterações neurológicas. Para diagnóstico definitivo é indispensável o exame histopatológico e nele se geralmente é observado intensa mitose, pleomorfismo e necrose extensas. Ainda não existe um protocolo padrão para tratamento do neuroblastoma olfatório, porém a remoção do tumor seguida de radioterapia é a modalidade de tratamento que tem dado maior tempo de sobrevida aos pacientes. Objetivou-se com este trabalho, relatar um caso de neuroblastoma olfatório em um cão sem raça definida com onze anos de idade destacando os métodos diagnósticos para esta doença, com ênfase nos achados tomográficos e histopatológico.

Palavras chave: clínica veterinária, neoplasia intranasal, tomografia computadorizada

\section{Olfactory neuroblastoma in dogs: tomographic and histopathological findings}

ABSTRACT. The occurrences of neoplasia's in the nasal cavity in dogs occur in approximately $1 \%$ of all neoplasms. Olfactory neuroblastoma is a neuroepithelial neoplasm of the nasal cavity and paranasal sinuses rarely reported in dogs and has as main clinical signs epistaxis, sneezing, respiratory distress, facial deformity and neurological changes. For definitive diagnosis, histopathological examination is indispensable, and extensive mitosis, pleomorphism and necrosis are generally observed. There is still no standard protocol for the treatment of olfactory neuroblastoma, but removal of the tumor followed by radiotherapy is the treatment modality that has given the patients a longer survival time. The objective of this work was to report a case of olfactory neuroblastoma in a dog with no defined breed at eleven years old, highlighting the diagnostic methods for this disease, with emphasis on tomographic and histopathological findings.

Keywords: veterinary clinic, intranasal neoplasm, computed tomography 


\title{
Neuroblastoma olfatorio en perro: hallazgos tomográficos e histopatológicos
}

\begin{abstract}
RESUMEN. Las frecuencia de neoplasias en la cavidad nasal en perros ocurren en aproximadamente el $1 \%$ de todas las neoplasias. El neuroblastoma olfatorio es una neoplasia neuroepitelial de la cavidad nasal y senos paranasales raramente relatada en perros y tiene como principales signos clínicos epistaxis, estornudos, dificultad respiratoria, deformidad facial y alteraciones neurológicas. Para el diagnóstico definitivo es indispensable el examen histopatológico y en él se suele observar una intensa mitosis, pleomorfismo y necrosis extensas. No existe un protocolo estándar para el tratamiento del neuroblastoma olfatorio, pero la remoción del tumor seguida de radioterapia es la modalidad de tratamiento que ha dado mayor tiempo de supervivencia a los pacientes. Se objetivó con este trabajo, relatar un caso de neuroblastoma olfatorio en un perro sin raza definida con once años de edad destacando los métodos diagnósticos para esta enfermedad, con énfasis en los hallazgos tomográficos e histopatológico.
\end{abstract}

Palabras clave: clínica veterinaria, neoplasia intranasal, tomografía computarizada

\section{Introdução}

O termo neoplasia significa massa de tecido anormal de crescimento não coordenado e excedente em relação ao tecido normal e persiste em crescer da mesma maneira após cessar o estimulo que causou sua mudança. Câncer é um termo comum utilizado para todas as neoplasias malignas e considerada uma doença multifatorial (Daleck et al. 2016).

As neoplasias estão entre as principais doenças que mais acometem os cães e gatos e são um dos principais causadores de óbito nestes animais (Sprenger et al. 2015). A incidência de cães diagnosticados com neoplasia está aumentando cada vez mais, tal fato deve-se a várias razões, entre elas está a maior longevidade observada nestes animais. Fatores associados como a nutrição e dieta mais balanceada, prevenção de doenças infectocontagiosas com vacinas, métodos de diagnósticos mais precisos e protocolos terapêuticos mais eficazes contribuem para maior longevidade dos cães (Priebe et al. 2011).

As neoplasias são classificadas em malignas e benignas estas não resultam em elevada mortalidade, não invadem tecidos vizinhos e não causam metástase, em contrapartida, as primeiras causam maior destruição tecidual e geralmente levam o animal a óbito (Dias et al. 2015; Pazzini et al. 2015). Neoplasias de cavidade nasal são consideradas raras e pouco descritas na literatura (Gonçalves et al. 2012) correspondem a $1 \%$ de todas as neoplasias em cães e cerca de $80 \%$ são malignas (Formighieri et al. 2012; Pazzini et al. 2015; Daleck et al. 2016). Elas podem ter origem em qualquer tipo de tecido incluindo osso, cartilagem, tecido conjuntivo, vasos sanguíneos e todos os diferentes tipos de células das glândulas e do epitélio de revestimento nasal (Formighieri et al. 2012; Zachary et al. 2012).

As neoplasias intranasais geralmente acometem animais de médio a grande porte e com idade média de 10 anos (Daleck et al. 2016; Pavelski et al. 2016). Alguns autores descrevem maior predisposição em cães dolicocefálicos que vivem em regiões urbanas devido ao contato da mucosa nasal com poluentes dispersados no ambiente (Pazzini et al. 2015; Daleck et al. 2016). Segundo Pazzini et al. (2015), animais expostos por longos período a fumaça de cigarro, inseticidas tópicos e a fumaça de aquecedor a querosene foi associado ao aumento de risco de desenvolvimento de carcinomas intranasais. As raças mais comumente descritas com risco de desenvolver a doença incluem Basset Hound, Old English Sheepdog, Scottish Terrir, Collie, PastorAlemão, Setter Irlandês, Pointer Alemão, Labrador, Staffordshire, Rottweiler, Bull Terrier e West Highland White Terrir (Daleck et al. 2016).

As neoplasias mais comuns na região nasal são adenocarcinoma, carcinoma de células escamosas e carcinomas indiferenciados (Formighieri et al. 2012; Nelson \& Couto 2015; Daleck et al. 2016; Pavelski et al. 2016) seguido do fibrossarcoma, condrossarcoma e osteossarcoma. Todos apresentam características de crescimento rápido e invasão local (Pavelski et al. 2016), as metástases são poucos frequente (Daleck et al. 2016). Tumores benignos incluem adenomas, fibromas, papilomas e tumores venéreos transmissíveis (Nelson \& Couto 2015). A super expressão da proteína p53 mutada e da 
ciclooxigenase 2 foi detectada nos tumores epiteliais da cavidade nasal (Daleck et al. 2016).

O neuroblastoma olfatório, também conhecido como estesioneuroblastoma, é uma neoplasia rara da cavidade nasal e seios paranasais de origem neuroepitelial que tem curso clínico imprevisível (Lubojemska et al. 2016). Os sinais clínicos que podem estar presentes em animais com neoplasia nasal são secreção nasal, espirros, dispneia, ruídos nasais, assimetria facial e convulsões nos casos em que a porção caudal da cavidade é afetada (Pazzini et al. 2015; Daleck et al. 2016; Pavelski et al. 2016). O crescimento da neoplasia para dentro da órbita pode causar exoftalmia ou inabilidade na retropulsão bulbar (Nelson \& Couto 2015).

O diagnóstico de neoplasia é feito por meio da associação de exames de imagem como a radiografia, tomografia computadorizada, ressonância magnética e rinoscopia e a análise histopatológica se faz necessário para diagnóstico definitivo (Pazzini et al. 2015). Antes do exame histopatológico, deve ser realizada a avaliação geral do paciente com anamnese, exame físico e exames complementares detalhados (Daleck et al. 2016). O exame radiográfico é o método mais frequentemente utilizado na medicina veterinária para verificar invasão óssea de neoplasias de tecido moles, porém não permite determinar com precisão a extensão tumoral e o envolvimento de tecidos adjacentes da neoplasia (Pavelski et al. 2016). No estágio inicial da doença, o exame de radiografia pode apresentar aspecto similar aos de um processo infeccioso. No geral, a radiografia pode mostrar aumento da densidade de tecido mole em região de osso nasal ou maxilar, destruição dos turbinados assimétricos, lise dos ossos faciais e opacificação do seio frontal (Daleck et al. 2016). A tomografia computadorizada é o exame de imagem que supre as limitações da radiografia, pois não ocasiona sobreposição de imagens melhorando a visibilidade da localização, extensão e severidade da enfermidade (Pavelski et al. 2016). Também é útil no planejamento cirúrgico, para avaliação de envolvimento ósseo e do acometimento da placa cribiforme, que indica invasão neoplásica para o sistema nervoso central (Daleck et al. 2016).

A rinoscopia é uma ferramenta bastante utilizada, porém deve ser utilizada para visualização do tumor. A citologia por escova ou lavado nasal não deve ser utilizado como ferramenta única no diagnóstico, já que muitas neoplasias têm baixa celularidade, comprometendo o resultado final (Daleck et al. $\underline{2016) .}$.

O tratamento consiste na associação de técnicas como a cirurgia, a radioterapia, a quimioterapia e a utilização da COX-2, contudo vai depender da localização e tamanho da neoplasia. As neoplasias intranasais apresentam comportamento local bastante agressivo, por isso seu tratamento deve objetivar, principalmente, o controle local da doença (Pazzini et al. 2015). Os glicocorticoides têm mostrado melhoria dos sinais clínicos (Nelson \& Couto 2015). Em humanos o tratamento com radioterapia alcançou uma excelente taxa de controle com toxicidade aceitável (Mori et al. 2015). Segundo Daleck et al. (2016), o uso da carboplatina com epirrubicina foram utilizados em um cão alcançando sobrevida de 134 dias. Piroxacam na dose de $0,3 \mathrm{mg} / \mathrm{kg}$ associado à carboplatina $300 \mathrm{mg} / \mathrm{m}^{2}$ também foram relatados em cães com carcinomas epidermoides de seio frontal e protocolo de doxorrubicina alternada com carboplatina e piroxicam proporciona em média 210 dias de sobrevida. Também são relatos tratamentos com fluoruracila e ciclofosfamida, mitoxantrona, rinotomia e curetagem transnasal; porém todos têm resultados pobres em comparação à radioterapia.

O prognóstico de animais diagnosticados com neoplasias nasal é ruim, tendo em vista que a doença geralmente manifesta-se em estadiamento avançado e localizado em uma região que impossibilita a remoção cirúrgica. A presença de epistaxe pode indicar pior prognóstico (Nelson \& Couto 2015; Daleck et al. 2016). A eutanásia é frequentemente requisitada em decorrência de persistência da epistaxe, respiração dificultada, anorexia e perda de peso ou sinais neurológicos (Nelson \& Couto 2015).

O objetivo deste trabalho foi descrever um relato de caso de neuroblastoma olfatório em canino, assunto ainda pouco difundido na literatura especializada, além de também fornecer informações acerca dos sinais clínicos, achados mais relatos, prognóstico e tratamento afim de se contribuir com a literatura.

\section{Relato de caso}

Um cão sem raça definida, fêmea com onze anos de idade foi atendida em uma clínica veterinária com histórico de deformidade facial com evolução há dois meses, epistaxe, respiração pela boca e perda de peso. Animal era vacinado, 
tinha uma dieta a base de ração super premium e morava em um centro urbano. Ao exame físico foi constatado aumento na região nasal, animal taquipneico e magreza. Foi realizado exames de rotina e solicitada a tomografia computadorizada em busca da extensão da massa. Também foi solicitado exame histopatológico pela técnica de biopsia incisional com o objetivo de obter diagnóstico definitivo. Foi prescrito tratamento paliativo, com anti-inflamatório não esteroidais e analgésicos, pelo grande comprometimento da neoplasia em cavidade nasal impossibilitando a ressecção cirúrgica com margens de segurança e devido ao difícil acesso à radioterapia, tendo em visto que é o tratamento de eleição nesses casos.

\section{Resultados e Discussão}

Em relação a predisposição do neuroblastoma olfatório, a literatura relata que a maioria dos animais acometidos tem mais de 5 anos de idade e os principais sinais clínicos que podem estar presentes são secreção nasal, espirros, dispneia, assimetria facial (Figura 1) e convulsão quando a massa atinge a porção caudal da cavidade (Pazzini et al. 2015; Pavelski et al. 2016) o que corrobora com os sinais clínicos apresentados pelo animal do presente relato, havia secreção nasal, espirros, dispneia, assimetria facial e como a neoplasia não tinha ultrapassado a placa cribriforme, $o$ animal do presente relato não apresentada convulsão.

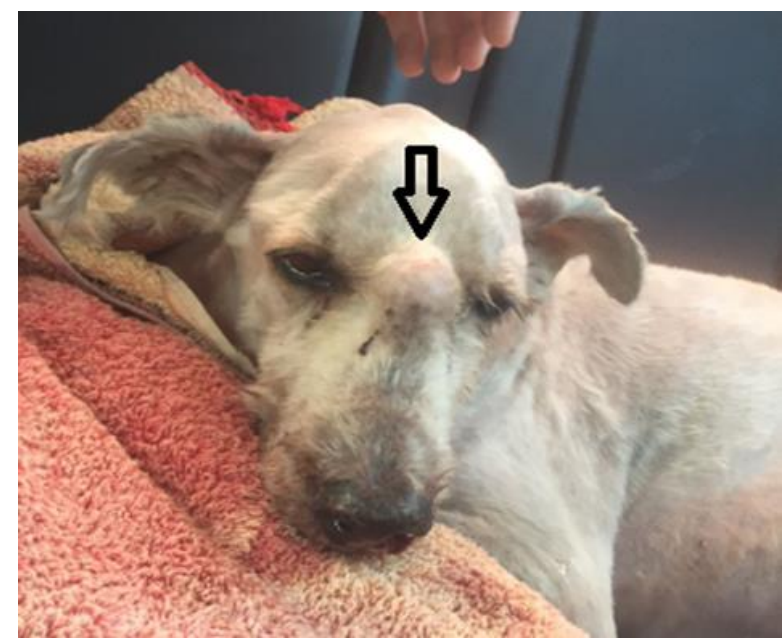

Figura 1. Foto demonstrativa da assimetria facial em região nasal.

Para melhor visualização da extensão da massa e para um possível planejamento cirúrgico foi realizado o exame tomográfico que visualizou presença de massa de grandes dimensões envolvendo cavidade nasal esquerda (Figura 2), com obstrução total da passagem do ar. Houve uma extensão da lesão para cavidade nasal direita, com destruição total de septo nasal e estendendose caudalmente para região de nasofaringe e seios frontais (Figura 3). Apresentou deformação em região de osso nasal e presença de importante destruição de turbinados nasais. A massa tinha aspecto heterogêneo, com atenuação radiográfica de tecidos moles. Após administração de contraste iodado intravenoso observou-se importante impregnação de contraste, com presença de áreas hipocaptantes e captação em região de seio frontal direito e região de nasofaringe. Havia importante lise do osso nasal, frontal, maxilar, zigomático, palato duro e orbita esquerda. Parênquima encefálico adjacente estava com aspecto tomográfico dentro da normalidade e linfonodos retrofaringeos com dimensões preservadas. Os achados tomográficos eram compatíveis com neoplasia intranasal, sendo sugerido como diagnóstico diferencial tumor de células mesenquimais e carcinoma de células escamosas.

Como diagnóstico diferencial para o neuroblastoma olfatório, devem ser considerados outros neoplasmas como o osteossarcoma, carcinoma de células escamosas, fibrossarcoma, hemangiossarcoma, condrossarcoma e afecções não neoplásicas da cavidade nasal como a rinite micótica por exemplo. No exame histopatológico realizado no presente relato, foi visto alta densidade celular, composta de células arredondadas a epitelioides, dispostas em arranjos sólidos e ocasionalmente com formação de estruturas semelhantes a rosetas. As células neoplásicas demonstraram núcleos arredondados de cromatina densa com discreta a moderada anisocariose com pequenos nucléolos ocasionalmente evidentes e citoplasma em moderada quantidade acidofilico. Observou cerca de 8 mitoses em 10 campos de 400x acompanhada de áreas coalescentes de hemorragia tumoral, concluindo ser compatível com o neuroblastoma olfatório ou estesioneuroblastoma. No presente relato, tratando-se de uma neoplasia maligna, com comprometimento importante aos tecidos peritumoral em cavidade nasal, a terapia de escolha segundo a literatura é a radioterapia (Daleck et al. 2016); porém, é um tratamento de difícil acesso. Sendo assim optou-se em fazer tratamento paliativo tendo em vista que esta neoplasma responde bem aos anti-inflamatório não esteroidais, então foi prescrito Previcox ${ }^{\circledR}$ (Firocoxib) $57 \mathrm{mg}$ S.I.D, Cronidor ${ }^{\circledR}$ (Tramal) T.I.D e Gaviz ${ }^{\circledR}$ (Omeprazol) 20mg S.I.D em jejum tendo melhora do quadro clínico. 

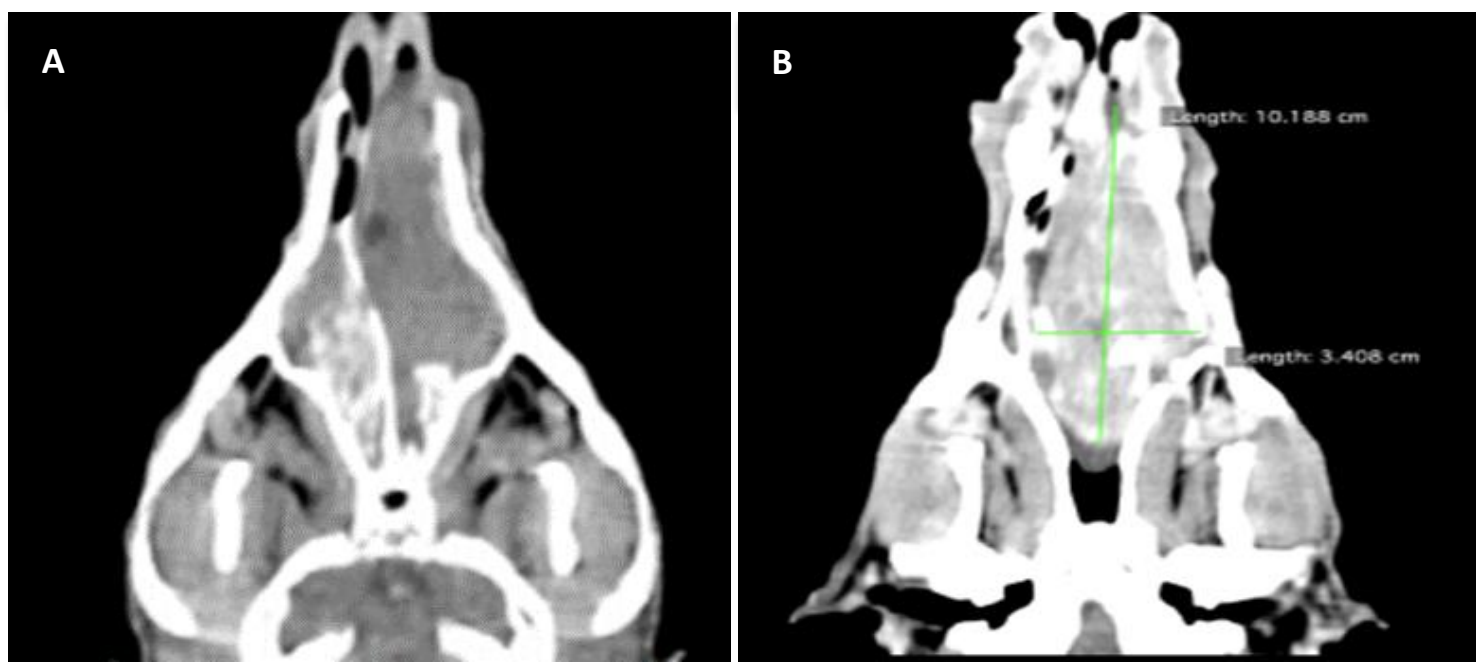

Figura 2. Exame tomográfico corte sagital (A) demonstrando massa de grande dimensão envolvendo cavidade nasal esquerda (B) Destruição total do septo nasal estendendo-se caudalmente para região de nasofaringe e seios frontais com medidas de $10,138 \mathrm{~cm}$ por $3,408 \mathrm{~cm}$.
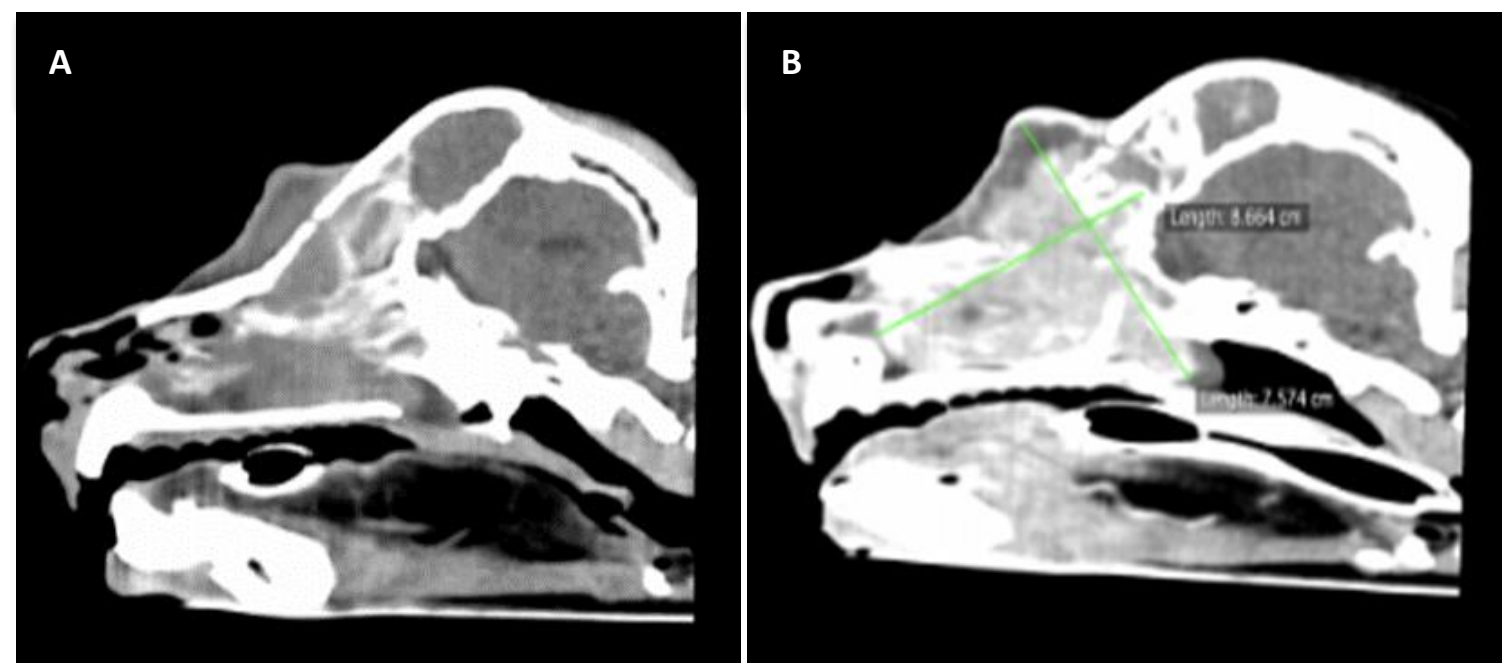

Figura 3. A). Demonstrando deformação em região de osso nasal. (B) Demonstrando medida da massa de 8,66 cm por $7,57 \mathrm{~cm}$.

\section{Conclusão}

Neoplasias intranasais se apresentam na grande maioria malignas e devem sempre constar na lista de diagnóstico diferenciais em casos de alterações clínicas no sistema respiratórios superior, entre eles achados como epistaxe, espirros e assimetria facial, relatados em cães a partir de 5 anos de idade, pois o diagnóstico precoce garante maior chance de cura ou de maior tempo de sobrevida livre da doença.

\section{Referências Bibliográficas}

Daleck C.R., Fonseca C.S. \& Canola J.C. 2016. Oncologia em cães e gatos. Roca, Rio de Janeiro.

Dias F., Dias L., Pereira L., Cabrini T. \& Rocha J. 2015. Neoplasias orais nos animais de companhia-Revisão de literatura. Revista

científica eletrônica de medicina veterinária 20, 1-9.

Formighieri A.P., Cella A.C.C., Carvalho M.F., Andrioli L.G. \& Meirelles A.C.F. 2012. Adenocarcinoma nasal em cão: relato de caso. PUBVET 6, 1289-94.

Gonçalves M.A., Dutra L.F.C., Quevedo L.S., Schreiner T. \& Anjos B.L. 2012. Condrossarcoma primário de cavidade nasal em um cão. Acta Scientiae Veterinariae 40, 15.

Lubojemska A., Borejko M., Czapiewski P., Dziadziuszko R. \& Biernat W. 2016. Of mice and men: olfactory neuroblastoma among animals and humans. Veterinary and comparative oncology 14, 70-82.

Mori T., Onimaru R., Onodera S., Tsuchiya K., Yasuda K., Hatakeyama H., Kobayashi H., 
Terasaka S., Homma A. \& Shirato H. 2015. Olfactory neuroblastoma: the long-term outcome and late toxicity of multimodal therapy including radiotherapy based on treatment planning using computed tomography. Radiation oncology 10, 1-9.

Nelson R.W. \& Couto C.G. 2015. Medicina interna de pequenos animais. Elsevier Editora, Amsterdan.

Pavelski M., Silva D.M. \& Froes T.R. 2016. Radiografia das cavidades craniana e nasal em afecções neoplásicas em cães: características e limitações. Veterinária e Zootecnia 23, 16473.

Pazzini J.M., De Nardi A.B., Serafim E.L., Calazans S.G., Huppes R.R., Rocha C.E.B.S., Viéra R.B., Oliva C.A.C., Bueno C. \& Marchiori M.G.A.F. 2015. Rinotomia dorsal empregada no tratamento de carcinoma intranasal em dois cães Rhinotomy backbone in treatment in two dogs intranasal carcinoma. Revista Portuguesa de Ciências Veterinárias 110, 240-5.
Priebe A.P.S., Riet-Correa G., Paredes L.J.A., Costa M.S.F., Silva C.D.C. \& Almeida M.B. 2011. Ocorrência de neoplasias em cães e gatos da mesorregião metropolitana de Belém, PA entre 2005 e 2010. Arquivo Brasileiro de Medicina Veterinária e Zootecnia 63, 1583-6.

Sprenger L.K., Risolia L.W., Gabardo L., Molento M.B., Silva A.W.C. \& Sousa R.S. 2015. Tumores neoplásicos de cães e gatos diagnosticados no laboratório de patologia veterinária da Universidade Federal do Paraná. Archives of Veterinary Science 20, 10-6.

Zachary J.F., McGavin D. \& McGavin M.D. 2012. Bases da patologia em veterinária. Elsevier Brasil, Rio de Janeiro.

Recebido: 13 mai. 2018

Aprovado: 11 jun. 2018

Publicado: 11 jul. 2018

Licenciamento: Este artigo é publicado na modalidade Acesso Aberto sob a licença Creative Commons Atribuição 4.0 (CC-BY 4.0), a qual permite uso irrestrito, distribuição, reprodução em qualquer meio, desde que o autor e a fonte sejam devidamente creditados. 